\title{
Colorectal Surgery in COVID-Negative Patients in the Early Phases of the Pandemic: Short-Term Outcomes
}

\author{
Sarah B. Stringfield ${ }^{1}\left[0\right.$. Gerald O. Ogola ${ }^{2} \cdot$ Rachel Curran $^{1} \cdot$ Katerina O. Wells ${ }^{1} \cdot$ Alessandro Fichera $^{1}$. \\ James W. Fleshman ${ }^{1}$
}

Received: 30 April 2021 / Accepted: 2 August 2021 / Published online: 17 August 2021

(c) The Society for Surgery of the Alimentary Tract 2021

Keywords COVID-19 $\cdot$ Colorectal surgery $\cdot$ Enhanced Recovery after Surgery $\cdot$ Ileus

\section{Introduction}

Many elective operations were canceled early in the COVID19 pandemic in order to conserve resources and to keep patients out of public settings. Our institution canceled elective cases except those that were considered "medically necessary to correct a serious medical condition or to preserve the life of a patient ${ }^{1}$." For colorectal patients, this included emergencies and selected cases such as cancer resections. We noticed that a high number of patients that underwent surgery during this time developed postoperative ileus (POI) and had a prolonged hospital stay. Our hypothesis was that patients undergoing surgery during the pandemic had worse short-term outcomes, despite being COVID-negative.

\section{Materials and methods}

This is a retrospective review of COVID-19 negative patients undergoing elective abdominal colorectal surgery at Baylor University Medical Center in Dallas, Texas. They were divided into those that underwent surgery early in the pandemic when elective cases were canceled (3/16-5/3/2020) and the first month after resumption of elective cases (5/4-31/2020). Control group included patients that underwent surgery 1 year prior (3/1-4/30/2019). Operations were considered elective if the patient was admitted from home for the operation, and not through the Emergency

Sarah B. Stringfield

sarah.stringfield@bswhealth.org

1 Department of Surgery, Baylor University Medical Center, 3500 Gaston Ave, Dallas, TX 75246, USA

2 Baylor Scott \& White Research Institute, 3310 Live Oak Street, Ste 501, Dallas, TX 75204, USA
Department or transfer from outside hospital. Nasogastric tube (NGT) insertion and need for TPN was excluded if present preoperatively or placed in the operating room.

\section{Results}

When analyzing all the patients, we found differences in sex, indication for surgery, and operative time, but not other demographics (Table 1). There continued to be differences in NGT insertion and need for postoperative TPN, resulting in longer time to a solid diet and LOS. The highest rates of these outcomes were found in the MarApr20 group.

Elective cases performed in 2020 were case-matched 1:1 to 2019 cases based on age, sex, and type of operation. There continued to be statistically higher rates of need for TPN, NGT insertion, and LOS in the patients that underwent surgery in MarApr20 (Table 2). Time to tolerating a diet was no longer significant.

\section{Discussion}

When elective cases were canceled early in the pandemic, COVID-19 negative patients that underwent surgery had higher rates of POI. We believe a large factor in the worse outcomes is a reflection of poor adherence to Enhanced Recovery After Surgery (ERAS) protocols. From March to May, the ERAS coordinator could not provide in-person education to patients. Patients were relocated to another surgical floor while the colorectal floor was converted into a COVID unit, so patients likely did not receive the same care that is typically provided by nurses experienced with ERAS. Patients were also not encouraged to leave their rooms to ambulate in the hallways. 
Table 1 Patient characteristics - all elective cases

\begin{tabular}{|c|c|c|c|c|}
\hline & $\begin{array}{l}\text { Control } \\
\text { MarApr } 2019 \\
(\mathrm{~N}=83)\end{array}$ & $\begin{array}{l}\text { MarApr } \\
2020 \\
(\mathrm{~N}=11)\end{array}$ & $\begin{array}{l}\text { May } \\
2020 \\
(\mathrm{~N}=33)\end{array}$ & P-value \\
\hline Age (years) & $56.4(17.0)$ & $55.8(16.4)$ & $57.9(17.6)$ & 0.898 \\
\hline Sex & & & & $0.012 *$ \\
\hline Male & $36(43.4 \%)$ & $9(81.8 \%)$ & $10(30.3 \%)$ & \\
\hline Female & $47(56.6 \%)$ & $2(18.2 \%)$ & $23(69.7 \%)$ & \\
\hline $\begin{array}{l}\text { American Society of Anesthesi- } \\
\text { ologists (ASA) class }\end{array}$ & & & & 0.142 \\
\hline 1 & $0(0.0 \%)$ & $0(0.0 \%)$ & $1(3.0 \%)$ & \\
\hline 2 & $40(48.2 \%)$ & $4(36.4 \%)$ & $10(30.3 \%)$ & \\
\hline 3 & $38(45.8 \%)$ & $7(63.6 \%)$ & $22(66.7 \%)$ & \\
\hline 4 & $5(6.0 \%)$ & $0(0.0 \%)$ & $0(0.0 \%)$ & \\
\hline Type of operation & & & & 0.396 \\
\hline Minimally Invasive & $54(65.1 \%)$ & $7(63.6 \%)$ & $17(51.5 \%)$ & \\
\hline Open & $29(34.9 \%)$ & $4(36.4 \%)$ & $16(48.5 \%)$ & \\
\hline Indication & & & & $0.006^{*}$ \\
\hline Benign & $29(34.9 \%)$ & $1(9.1 \%)$ & $17(51.5 \%)$ & \\
\hline Cancer & $34(41.0 \%)$ & $10(90.9 \%)$ & $9(27.3 \%)$ & \\
\hline Inflammatory Bowel Disease & $20(24.1 \%)$ & $0(0.0 \%)$ & $6(18.2 \%)$ & \\
\hline Ileostomy & $0(0.0 \%)$ & $0(0.0 \%)$ & $1(3.0 \%)$ & \\
\hline Operative time (hours) & $4.0(2.6,4.9)$ & $3.6(2.2,6.9)$ & $2.6(2.0,3.5)$ & $0.039 *$ \\
\hline EBL $(\mathbf{m L})$ & $50.0(5.0,100.0)$ & $25.0(10.0,75.0)$ & $50.0(0.0,100.0)$ & 0.756 \\
\hline LOS (days) & $3.0(2.3,4.0)$ & $5.0(3.6,8.4)$ & $3.0(2.3,4.0)$ & $0.030 *$ \\
\hline TPN & $0(0.0 \%)$ & $2(18.2 \%)$ & $1(3.0 \%)$ & $<0.001^{*}$ \\
\hline NGT inserted & $4(4.8 \%)$ & $6(54.5 \%)$ & $4(12.1 \%)$ & $<0.001 *$ \\
\hline Time to solid diet (days) & $1.7(0.9,2.7)$ & $3.7(1.9,6.4)$ & $1.8(0.9,2.8)$ & $0.034 *$ \\
\hline 30-day Readmission & $8(9.6 \%)$ & $1(9.1 \%)$ & $4(12.1 \%)$ & 0.916 \\
\hline Complications (Clavien-Dindo) & & & & 0.204 \\
\hline Grade 2 & $10(71.4 \%)$ & $3(60 \%)$ & $3(42.9 \%)$ & \\
\hline Grade 3 & $4(28.6 \%)$ & $2(40 \%)$ & $3(42.9 \%)$ & \\
\hline Grade 4 & $0(0 \%)$ & $0(0 \%)$ & $0(0 \%)$ & \\
\hline Grade 5 & $0(0 \%)$ & $0(0 \%)$ & $1(14.3 \%)$ & \\
\hline \multicolumn{5}{|l|}{ Oral Morphine Equivalent use } \\
\hline First $24 \mathrm{~h}$ & $66.0(27.5,100.0)$ & $85.0(73.0,109.5)$ & $67.5(52.0,130.0)$ & 0.082 \\
\hline PODl to discharge & $22.5(0.0,73.7)$ & $24.0(0.0,90.2)$ & $34.0(7.5,58.0)$ & 0.654 \\
\hline OME/day & $33.6(13.5,54.0)$ & $29.8(18.3,43.8)$ & $33.0(23.9,50.4)$ & 0.503 \\
\hline
\end{tabular}

*p-value $<0.05$ is considered statistically significant
Stress is known to contribute to POI and may be an unmeasured factor with a significant impact. During March and April, our hospital did not allow visitors. Lack of support from friends or family negatively impacts patient experience, with patients less likely to receive timely receipt of medications and assistance getting out of bed ${ }^{2}$. While it was not directly measured in our patients, we observed that anxiety levels were highest early in the pandemic. Research surrounding mental well-being during the pandemic has found higher rates of anxiety and depression throughout the population ${ }^{3}$.
This study has several limitations, most importantly the fact that it is retrospective, with small numbers of highly selected patients in each group.

\section{Conclusion}

This study demonstrates the impact that adherence to an ERAS protocol, psychological support, and mental health has on patient outcomes, with even small changes having a potentially big impact. Reviewing patient outcomes leads 
Table 2 Matched analysis

\begin{tabular}{|c|c|c|c|c|}
\hline & $\begin{array}{l}\text { Control } \\
\text { MarApr } 2019 \\
(\mathrm{~N}=44)\end{array}$ & $\begin{array}{l}\text { MarApr } \\
2020 \\
(\mathrm{~N}=11)\end{array}$ & $\begin{array}{l}\text { May } \\
2020 \\
(\mathrm{~N}=33)\end{array}$ & P-value \\
\hline Age (years) & $55.9(18.2)$ & $55.8(16.4)$ & $57.9(17.6)$ & 0.870 \\
\hline Sex & & & & $0.011^{*}$ \\
\hline Male & $18(40.9 \%)$ & $9(81.8 \%)$ & $10(30.3 \%)$ & \\
\hline Female & $26(59.1 \%)$ & $2(18.2 \%)$ & $23(69.7 \%)$ & \\
\hline ASA class & & & & 0.812 \\
\hline 1 & $0(0.0 \%)$ & $0(0.0 \%)$ & $1(3.0 \%)$ & \\
\hline 2 & $16(36.4 \%)$ & $4(36.4 \%)$ & $10(30.3 \%)$ & \\
\hline 3 & $27(61.4 \%)$ & $7(63.6 \%)$ & $22(66.7 \%)$ & \\
\hline 4 & $1(2.3 \%)$ & $0(0.0 \%)$ & $0(0.0 \%)$ & \\
\hline Type of operation & & & & 0.783 \\
\hline Minimally Invasive & $24(54.5 \%)$ & $7(63.6 \%)$ & $17(51.5 \%)$ & \\
\hline Open & $20(45.5 \%)$ & $4(36.4 \%)$ & $16(48.5 \%)$ & \\
\hline Indication & & & & $0.016^{*}$ \\
\hline Benign & $16(36.4 \%)$ & $1(9.1 \%)$ & $17(51.5 \%)$ & \\
\hline Cancer & $19(43.2 \%)$ & $10(90.9 \%)$ & $9(27.3 \%)$ & \\
\hline Inflammatory Bowel Disease & $9(20.5 \%)$ & $0(0.0 \%)$ & $6(18.2 \%)$ & \\
\hline Ileostomy & $0(0.0 \%)$ & $0(0.0 \%)$ & $1(3.0 \%)$ & \\
\hline Operative time (hours) & $3.8(2.6,4.8)$ & $3.6(2.2,6.9)$ & $2.4(1.8,3.4)$ & $0.015^{*}$ \\
\hline EBL (mL) & $50.0(10.0,100.0)$ & $25.0(10.0,75.0)$ & $50.0(0.0,100.0)$ & 0.841 \\
\hline LOS (days) & $3.0(2.2,3.9)$ & $5.0(3.6,8.4)$ & $3.0(2.3,4.0)$ & $0.020^{*}$ \\
\hline TPN & $0(0.0 \%)$ & $2(18.2 \%)$ & $1(3.0 \%)$ & $0.012 *$ \\
\hline NGT inserted & $4(9.1 \%)$ & $6(54.5 \%)$ & $4(12.1 \%)$ & $<0.001^{*}$ \\
\hline Time to solid diet (days) & $1.7(0.9,2.3)$ & $3.7(1.9,6.4)$ & $1.8(0.9,2.8)$ & 0.055 \\
\hline 30-day Readmission & $5(11.4 \%)$ & $1(9.1 \%)$ & $4(12.1 \%)$ & 0.963 \\
\hline Complications & & & & 0.204 \\
\hline Grade 2 & $6(85.7 \%)$ & $3(60 \%)$ & $3(42.9 \%)$ & \\
\hline Grade 3 & $1(14.3 \%)$ & $2(40 \%)$ & $3(42.9 \%)$ & \\
\hline Grade 4 & $0(0 \%)$ & $0(0 \%)$ & $0(0 \%)$ & \\
\hline Grade 5 & $0(0 \%)$ & $0(0 \%)$ & $1(14.3 \%)$ & \\
\hline \multicolumn{5}{|c|}{ Oral Morphine Equivalent use } \\
\hline First $24 \mathrm{~h}$ & $66.0(30.0,109.6)$ & $85.0(73.0,109.5)$ & $67.5(52.0,130.0)$ & 0.166 \\
\hline PODl to discharge & $24.0(0.0,96.0)$ & $24.0(0.0,90.2)$ & $34.0(7.5,58.0)$ & 0.872 \\
\hline OME/day & $35.6(14.0,69.5)$ & $29.8(18.3,43.8)$ & $33.0(23.9,50.4)$ & 0.755 \\
\hline
\end{tabular}

*p-value $<0.05$ is considered statistically significant

to improved care in the future, during times of crisis as well as normalcy.

Author contribution SS designed the study, collected data, and wrote the manuscript. GO performed data analysis. RC collected data. KW, $\mathrm{AF}$, and JF provided study design and manuscript revisions. All authors read and approved the final manuscript.

\section{Declarations}

Ethics approval and consent to participate The Baylor University Medical Center IRB approved the study protocol. Owing to the observational, non-interventional study design, informed consent was waived.
Manuscript was presented as a Presentation on Demand for the 2021 American Society of Colon and Rectal Surgeons Annual Scientific Meeting, San Diego, CA (virtual), April 24-28, 2021.

Conflict of interest The authors have no conflicts of interest related to this study.

\section{References}

1. Abbott, Greg. Executive Order No. GA-09 relating to hospital capacity during the COVID-19 disaster. Online. EO-GA_09_ COVID-19_hospital_capacity_IMAGE_03-22-2020.pdf (texas. gov). Accessed 14 December 2020. 
2. [2] Zeh RD, Santry HP, Monsour C, Sumski AA, Bridges JFP, Tsung A, et al. (2020) Impact of visitor restriction rules on the postoperative experience of COVID-19 negative patients undergoing surgery. Surgery. 168(5):770-776.

3. [3] Wang C, Pan R, Wan X, Tan Y, Xu L, Ho CS, Ho RC. (2020) Immediate psychological responses and associated factors during the initial stage of the 2019 coronavirus disease (COVID-19) epidemic among the general population in China. Int J Environ Res Public Health. 17(5):1729.

Publisher's Note Springer Nature remains neutral with regard to jurisdictional claims in published maps and institutional affiliations. 